Please do not remove this page

RMIT

UNIVERSITY

\title{
A generalised CFD learning and prediction system
}

Becker, William; Tu, Jiyuan; Yu, Xinghuo

https://researchrepository.rmit.edu.au/esploro/outputs/9921858848701341/filesAndLinks?institution=61RMIT_INST\&index=null

Becker, W., Tu, J., \& Yu, X. (2007). A generalised CFD learning and prediction system. 5th IEEE International Conference on Industrial Informatics, 615-620. https://doi.org/10.1109/INDIN.2007.4384844

Published Version: https://doi.org/10.1109/INDIN.2007.4384844

Repository homepage: https://researchrepository.rmit.edu.au

(C) 2007 IEEE

Downloaded On 2023/04/26 21:18:58 +1000

Please do not remove this page 


\title{
A generalised CFD learning and prediction system
}

\author{
William Becker, Xinghuo Yu, Jiyuan Tu
}

\begin{abstract}
An expert system that learns data from Computational Fluid Dynamics (CFD) simulations and presents it to non-expert users of CFDs to make informed design decisions is proposed. The system is responsible for choosing CFDs to run, supervising their running, determining when to halt them and then teaching them to a Neural Network (NN). The system also includes a visualisation tool which displays the effect of CFDs on the output of the NN. A case study is finally presented to demonstrate how the tool has been used with fire simulations.
\end{abstract}

\section{INTRODUCTION}

The field of Computational Fluid Dynamics (CFD) is seldom coupled with Intelligent Systems (IS). However, there are now some compelling reasons to do so. CFDs are complicated systems and difficult to understand for untrained users. CFDs are being used in more everyday designs. For example, performance based building codes (based on simulations and experiments) are increasingly being used as design measures, as opposed to prescriptive codes [2]. The simplification of CFDs is thus becoming necessary: reducing the reliance on experts to simulate designs would reduce this hindrance to architects, designers and engineers in other fields. IS, in the form of expert systems, can be used as a facade to take CFDs out of the realm of CFD experts, by intelligently determining for users how simulations need to be run.

CFDs are a numerical method of estimating the velocities, pressures and temperatures of fluids over volumes, using the Navier-Stokes equations. They operate by splitting geometry in a 2 or 3 dimensions into a mesh of cells and solving the equations for each cell for a certain instant. They then increment the simulation time and solve the equations again. As the mesh is refined, the quality of the calculation is increased, however this also causes a commensurate increase in the running time. Thus, running times for CFDs may be in the order of months. Due to this, they are only practical for viewing the features of one-off instances and not useful for giving instant feedback to users. This also precludes them from being used as an input to an optimisation algorithm, such as the genetic algorithm, which requires many evaluations.

Some attempts have been made to use Intelligent Systems with CFDs over the past 20 years, although it is only with recent advances in hardware that such a coupling has become practical. Prior attempts have been two pronged: one avenue is to use Expert Systems (ES) to help the user generate the CFD (eg [3], [4]), the other involves using Neural Networks (NN) to learn data from CFDs (eg [6], [7], [10], [11]). These

W. Becker and J. Tu are with the School of Aerospace, Mechanical and Manufacturing Engineering, Royal Melbourne Institute of Technology,Bundoora, VIC 3083, Australia (email: s2006809@student.rmit.edu.au, jiyuan.tu@rmit.edu.au). X. Yu is with the School of Electrical and Computer Engineering, Royal Melbourne Institute of Technology, Melbourne, VIC 3000, Australia (email: x.yu@ rmit.edu.au).

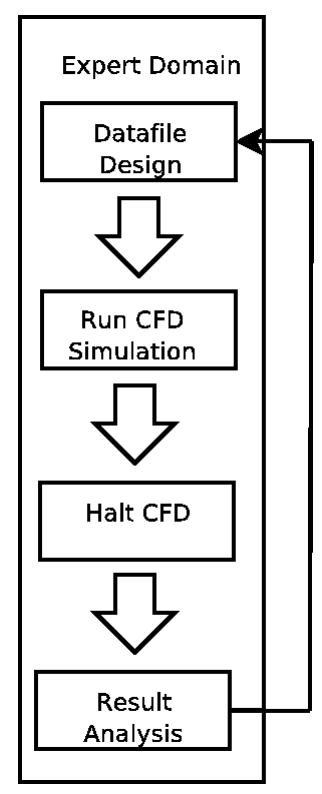

Fig. 1. CFD Creation Process

attempts have not eliminated the need for experts in most parts of the CFD running process, which would allow non-experts in the field such as architects and industrial designers to use their results.

There is a need to eliminate the barrier of entry to this field so that those who need to use the information gained from CFDs can do so without having to understand complicated fields of research, unrelated to their own. This paper therefore proposes creating a framework that separates the engineer's domain of creating and maintaining CFD simulations from the user's domain of analysing resultant information. This is achieved by automating the CFD process. The system, CFDLearner, deals with the entire procedure of CFD composition, learning and visualisation.

This paper first describes how CFDs are normally run, then shows how CFDLearner improves upon this process. The process of using CFDLearner is described and a case study is presented to show how CFDLearner has been used with a fire simulation system.

\section{UNDERLYING TECHNOLOGIES}

The proposed system is a fusion of three technologies, CFDs, Expert Systems and Neural Networks. The following section analyses their previous useage and highlights what deficiencies remain in known work.

\section{A. CFD usage}

To understand how the system works, it is important to know how CFDs are used. Figure 1 illustrates this. Firstly, the 


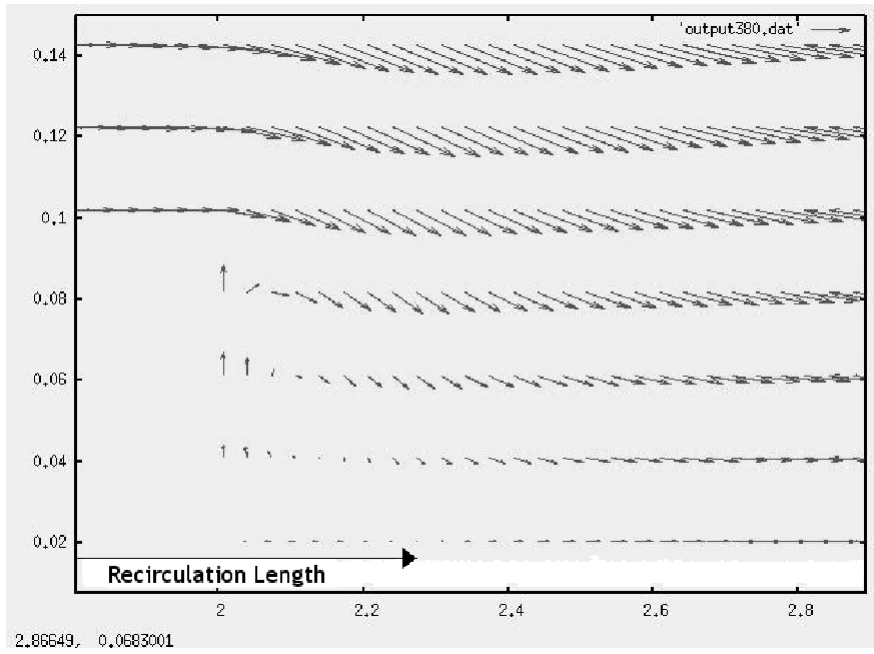

Fig. 2. Backwards Facing Step

simulation needs to be set up, which involves determining the geometry required, the initial conditions and the grid resolution for different parts of the volume. This is a difficult task and often requires a large amount of experimentation to perfect the environment required, especially for a novice user. It may be necessary to construct a datafile containing a large amount of hand coded data in a particular format.

Secondly, the CFD needs to be run. Running the CFD is generally not a problem, however knowing when to terminate the simulation may be. Prematurely stopping a CFD can result in the flow not having reached a stable state, whereas taking too long wastes computer resources and the user's time. It requires some amount of skill to properly find a balance point, as well as regular monitoring on the part of the user.

Thirdly, once the simulation is stopped, a result must be gleaned from the CFD. A CFD will generally provide output in the form of large arrays of various values, such as velocities in spatial dimensions, as well as pressures and temperatures. From these, some meaningful data is required. Maintaining the large arrays is not necessarily helpful, as it is relatively difficult to compare and optimise large datasets, especially across multiple experiments. Alternatively, it is simpler to try to extract parameters from the experiment. For example, in the Backward Facing Step problem (Figure 2), the focus is on the length of the recirculation region. This can be calculated by analysing the velocity array. Similarly other CFDs will have particular values which are characteristic of the simulation and useful in designs based upon them.

\section{B. Expert Systems}

Expert systems have been used in the past to try and ease the usage of CFD for normal users, by helping users decide on decisions that they need to make when running CFDs. In [3] a generalised system was designed to remove the need for users to create data files for CFDs. The system used an expert system to derive the information needed for their generation. This removed a great deal of work to understand the complex formats that CFDs generally need as input, which allowed beginning users to focus more on the actual simulations at hand. In contrast, Knight et al [4] used an expert system, FLOWES, to help create appropriate system parameters, eg varying the resolution of the grid to improve the speed and accuracy of a CFD.

Knight et al extends this research further [8] to help naive users design appropriate geometries, by using a Case-Based Reasoning (CBR) component. This attempted to formulate a way in which expertise could be incorporated at all stages of the modelling cycle. Their work reduced the time to set up and model a problem substantially. These same authors applied their work to the specific problem of fire modelling, which used CBR to help fire safety engineers design spatial layouts which were then simulated [5].

These systems are all intended to be "over the shoulder" helpers, guiding a user in producing good results, however they do not abstract away the actual CFD entirely. This is necessary for non-engineering specialists, such as architects or designers who are not concerned with the elicitation of results, only the use thereof.

\section{Neural Networks}

Neural Networks (NN) are systems designed to quickly predict data, interpolating and extrapolating from learning sets. Due to their speed, research has been performed to use them in the place of CFDs. For example, in [7], 72 CFD simulations over three variables, which each had a fixed amount of settings $(3,4$ and 6$)$ were run to train a neural network. The resulting 3 input $\mathrm{NN}$ was used for sensitivity analysis and to estimate the outputs for a gradient descent optimiser. On a larger scale, Tan et al [10], predicted the levels of a number of gases caused by burning coal. This data was then taught to a Multiple Level Perceptron (MLP) and optimised using Genetic Algorithms (GA). Similar work with NN and GA has been done in the fields of Fire Safety ([11], [6]). These systems, however, are all used by experts in the field of CFD and would not be appropriate for those unaccustomed to both CFDs and NNs.

\section{SySTEM OVERVIEW}

The CFDLearner system is a framework which automates the entire process of choosing which CFDs to run, running them and deciding when to halt them. It also extracts variables from the CFDs, teaches them to a learning system, determines when no further CFDs need to be run, and visualises the results. This allows user refinement of the results, which can then be used as an input to an optimisation tool or gives the user a deeper understanding of a complex dynamic system.

The system is implemented in the Java programming language and makes use of a modular Object Orientated framework. Its components have been designed so that they are independent and uncoupled, which allows trivial replacement with other modules that implement the same interfaces. Using this design, the active CFD can be easily changed, as well as other crucial parts of the algorithms.

From a user perspective, the proposed system has five steps to its usage: plugin design, system selection, CFD composition, CFD Learning and Result Visualisation (Figure 3). While 


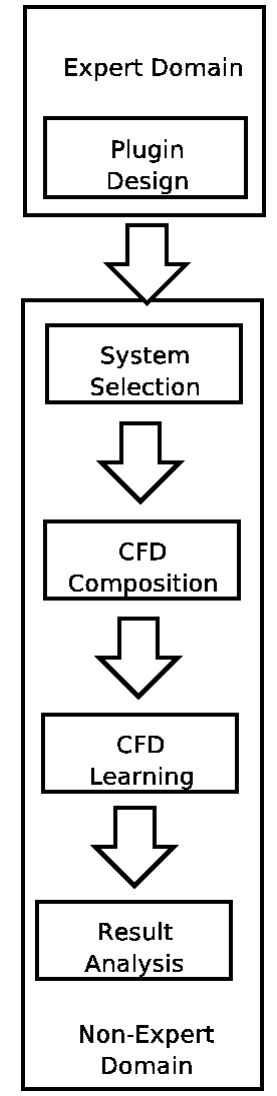

Fig. 3. System Flowchart

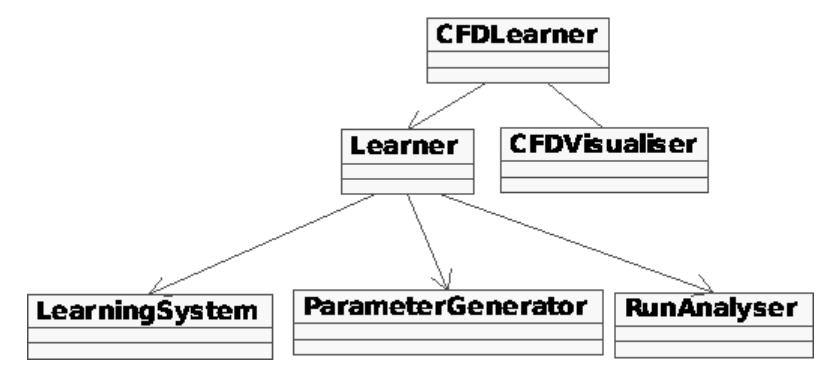

Fig. 4. System Overview

these are used sequentially, it is possible for individual parts of the system to be utilised by 3rd party applications. For example the NN could be used as an input to a CAD or optimisation tool, foregoing the visualisation stage.

An overview of the system in the form of a class diagram is shown in Figure 4. The main class is the CFDLearner, which has a Visualiser and a Learner. The learner initialises the system by loading data files which determine which CFD should be analysed, in addition to which components should be analysed. It then spawns the CFD and teaches the output to the learning system. It uses a ParameterGenerator to determine the parameters with which a CFD should be run and teaches these to a LearningSystem. The LearningSystem then uses these results to predict values based on inputs.

\section{A. Plugin Design}

The tool makes no assumptions about the CFD. Hence specific code needs to be written that determines how the system will interact with the particular CFD. This involves initialising the CFD, monitoring its progress, extracting data and analysing this data into meaningful information. This is the sole section that needs to be implemented by an expert in the field of CFD.

1) Learner: The Learner is responsible for training a neural network with the outputs of CFDs. It is designed to work on parallel systems and thus can spawn many RunAnalysers. Its purpose is twofold: initialisation of CFD specific data and training the neural network.

On initialisation from a data file it will determine the CFD, and hence the CFD plugins it should be using. It will then determine the parameters of the CFD plugin, which variables should be manipulated and which should be maintained. Particular parts of this are handled by the default learner, however sections pertinent to the specific CFD also must be overridden so appropriate variables for the CFD are loaded.

2) RunAnalyser: This module starts individual CFDs, monitors the progress of the CFD by evaluating theits output (so the system can determine whether it is stable), halts the CFD on reaching a quiescent state and finally extracts parameters from it. These steps are all CFD dependant and should be the focus of a 3rd party programmer developing a plugin.

Monitoring of the CFD: the user should take a snapshot of the CFD progress by observing a single instant of data or some averaged data over a short interval. The data is input to a filter, which detects when the CFD has reached a stable state. Since CFDs generally will not reach a stable state due to turbulent effects, it is necessary to determine if the CFD should be terminated with a degree of latitude. The filter accomplishes this by analysing the data and determining if a number of results are quite similar, within a given tolerance, asymptotically trending towards a value or are repeating in a cyclical manner.

When the CFD has reached a quiescent state, analysis parameters are extracted. These results are then taught to a learning system, such as a NN. If a quiescent state was not reached after a certain time or number of iterations, then the inputs of the CFD are noted and taught to another learning system. This system learns which sets of parameters are not likely to reach a steady state, so that similar CFDs will not be run in the future.

\section{B. System Selection}

If the relevant plugins are already developed, the system can be used by a non-expert. First it is necessary to properly initialise it and this means making some choices.

1) LearningSystem: This determines how the system will extrapolate or interpolate data. The best candidate to is a neural network (NN). Two kinds of NNs have been developed for used here: a variant of the GRNN (a kernel based NN), the GRNNFA+ [1] and a simple Multiple Layer Perceptron (MLP) NN. The GRNNFA+ has been specially developed for this purpose and learns and interpolates values quickly, while the MLP takes longer to train. 
2) ParameterGeneration: This determines the parameters that CFDs will be run from. Three kinds of parameter generation modules that been developed, however it is possible to extend these and add others. The three kinds are: Random, Ordered and Adaptive.

The random method picks random values for each parameter that needs to be chosen for the CFD. The ordered method selects values by choosing combinations of the extreme values for each parameter, then using combinations of middle and extreme values, etc. This suffers from the "curse of dimensionality", in that as the number of parameters increases, the number of different combinations increases at an exponential rate. To remedy this, an intelligent adaptive search method has been implemented, which tries to determine where the most information may be gained. This is done by determining which learned points have the largest difference between the output parameters and learning values that are between them.

\section{Composition}

CFD composition deals with the setting the input parameter range for the $\mathrm{CFD}$ and determing the desired outputs from those that are available from the plugin design. There may be several parameters to set for a particular CFD and the results may need to be compressed to be over a certain range.

A CFD will have a number of fixed variables and a number of dynamic variables. For instance, consider the example of designing a room from the perspective of fire safety. CFDs may simulate a fire in the room and the temperatures in the room may be measured. In this case there are many things that can vary: the size of the room, the number and location of openings, the position, fuel, size and strength of the fire. Attempting to train a system using with more than ten variables will suffer the "curse of dimensionality": to generate each combination of outlier results will require $2^{10}$ evaluations, which will take an inordinate amount of time using CFDs. Instead, fewer variables should be used.

\section{Learning}

Given settings, the system learns data from the CFD by evaluating the parameters of the runs to be performed, performing these runs and teaching data to a learning system.

The other responsibility of the Learner is to run CFDs, with which the LearningSystem is trained. Since the system runs may operate on parallel or sequential systems, it must determine whether enough CFDs are being run, to fill its allocated quota. It can use the PBS scheduling system to determine how many processors are available on the system and utilise spare resources. It may be network friendly, and yield to other processes that are queued. It may also run a single CFD run over multiple processors. If spare resources exist for a CFD, it determines which parmeters are to be used the from ParameterGenerator. With these parameters, a RunAnalyser is spawned which will control and monitor the CFD. The results gained from the CFD are then taught to the LearningSystem.

The Learner maintains a record of all CFDs that have been previously run and are currently run to prevent redundant

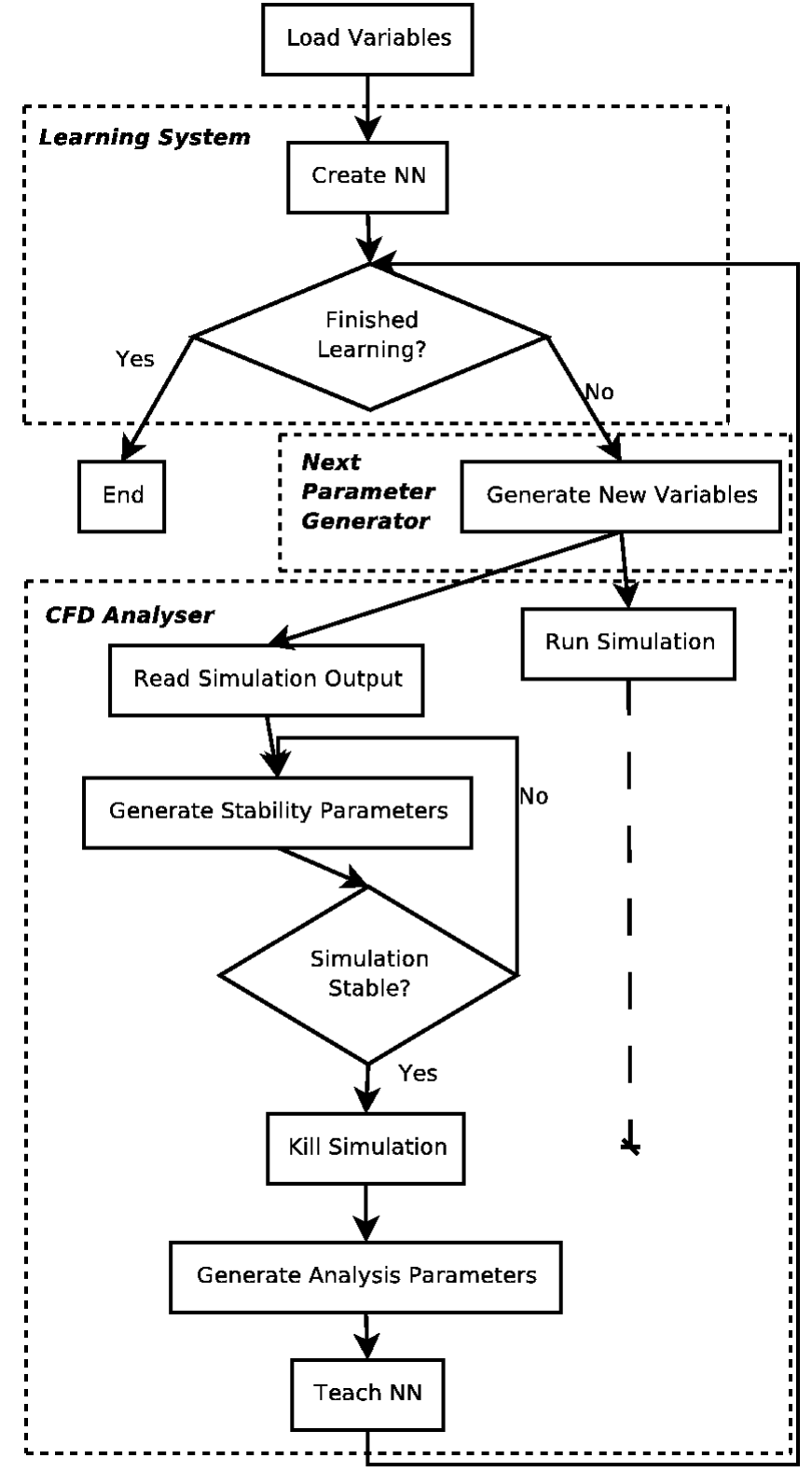

Fig. 5. Training Method

simulations from being invoked. This allows users to visualise previous simulations and allows an evaluation of the accuracy of predictions based on how much training data exists.

\section{E. Visualisation}

The visualisation tool interrogates the learner and displays the variables that have been taught, the predictions of the learner and allows for additional CFD runs to be performed.

The interface is shown in Figure 8. The bottom left window plots two variables from the output of the learner, with all other parameters held constant. The $\mathrm{x}$ and $\mathrm{y}$ axes are chosen on the panel on the right. Above the output are shown the actual locations of CFD runs relative to the viewed output. Both locations and learner output are rotatable and scaleable. When a transform occurs on one graph, it also is applied to the other, so that the views are synchronised. 


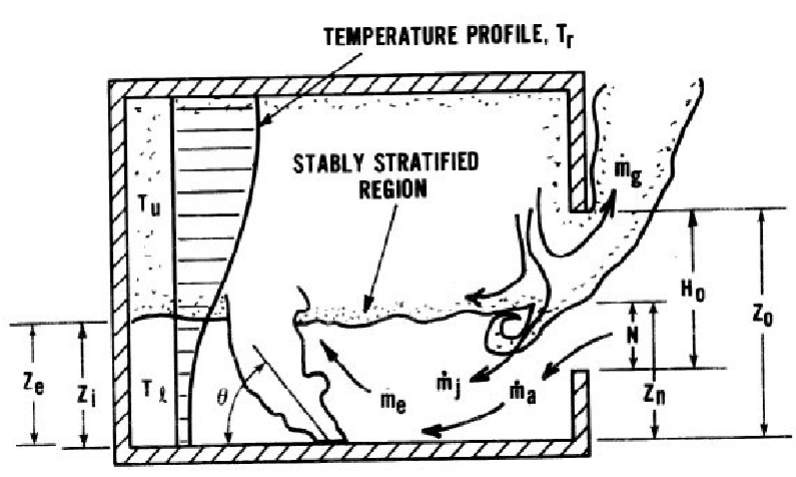

Fig. 6. Steckler's Experiment

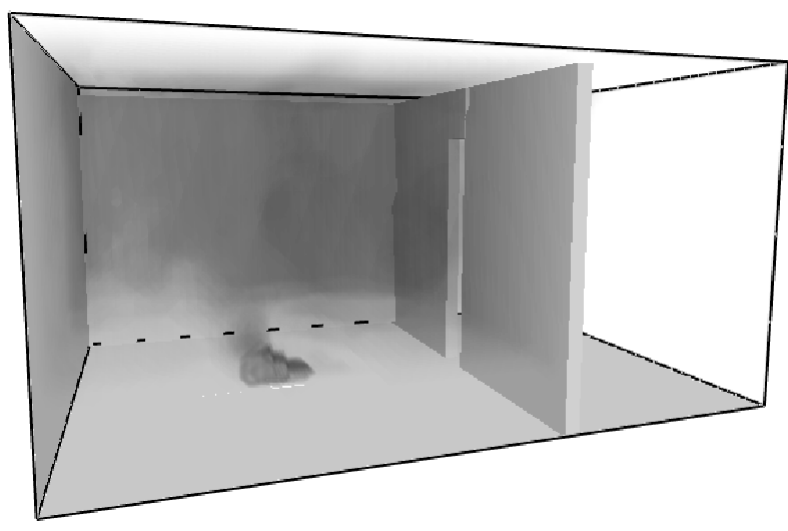

Fig. 7. Simulated Experiment

Since only two parameters may be plotted, the other parameters are set as a constant. Each such variable may be manipulated by means of a slider bar; the learner output will correspondingly change. This, however, results in a difference between the shown learner output and the CFD run parameters viewed in the upper left hand window. To show the correlation between the CFD run with respect to the other static run parameters, the size of the cube representing the run is altered. As the parameters more closely represent values that have been taught to the NN, the size of the cube increases, Having results in the vicinity of such points means that there is a higher degree of certainty in the results.

\section{RESULTS}

The system was constructed for usage with NIST's FDS, the Fire Dynamics Simulator. It is used to simulate fires in buildings and in this case was used to replicate the experiments done by Steckler, et al [9].

The plugin for the FDS CFDs loads extra variables required (such as the geometry of the room and the type of material to be burned) and managed the execution of the simulation. The RunAnalyser for each simulation created a file for the CFD process, but was not started. The FDS does not have an output mechanism that allows for fine grained control of the rate at which it outputs data about the simulation. Whilst analysing

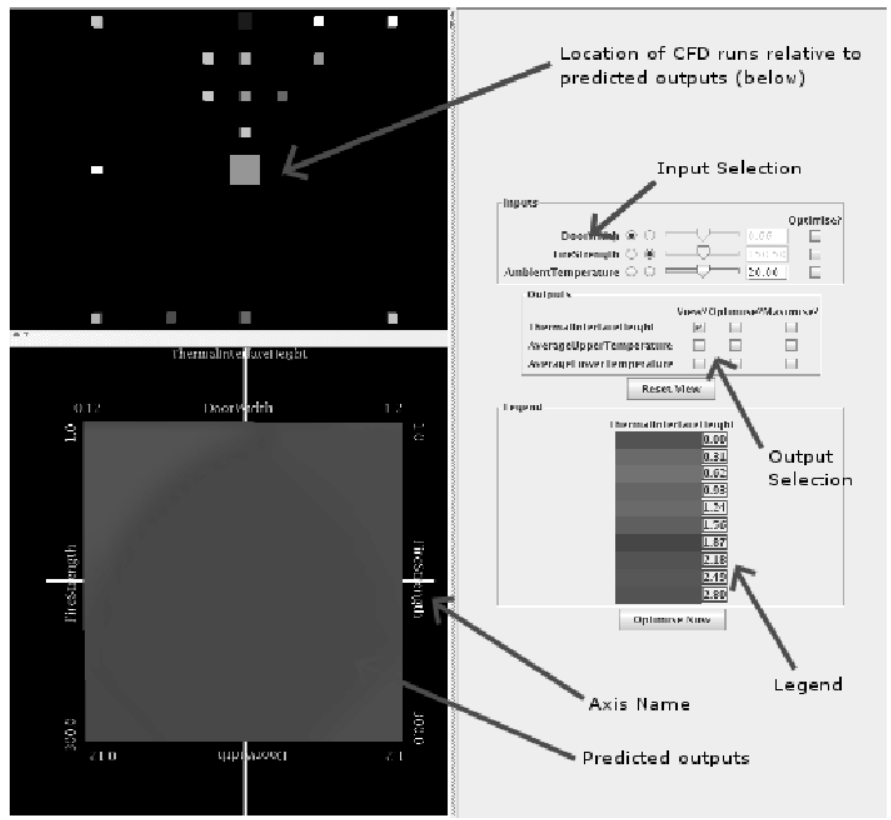

Fig. 8. Visualisation Tool

the data, it was found that averaging 10 results taken over 1 second of simulation time produced best results. However, if this amount of data was created thoughout the simulation it would have used too much storage space and slowed the simulation by spending an inordinate amount of time creating such output files. Instead when generating stability parameters, the simulation was run for 19 seconds of simulation time, without producing any output. After this it would stop and be restarted, having changed the data file to make the CFD output every 0.1 seconds of simulation time for 1 second. For each of the 10 results, the temperature in the corners of the room was extracted, as well as the flow rate out of the opening. These were used as they were same as the measurements taken by Steckler. It was then possible to compare the results and ensure the accuracy of the simulation.

When the error between successive results was within $10 \%$, analysis parameters were taken. This included determining the Thermal Interface Layer Height, and the temperature of the upper and lower layers (as shown in Figure 6). The system was selected to run with adaptive learning with the GRNNFA+ neural network and allowed to run on a 7 node cluster, with 2 processors per node. Then the results were viewed with the visualiser and analysed.

As shown in Figure 8, the trained NN gives an output that is viewable in the bottom left. Note that while 3 parameters were learned, only 2 are displayed. The other's value is adjustable by the drag bar in the input selection region. From this the viewer can receive real-time feedback about the region of the search space they have requested. This gives the potential to make quick design decisions about the case in question.

\section{Conclusion}

CFDs are difficult for the non-expert to run and analyse. CFDLearner resolves this by removing the difficulty of run- 
ning and analysing CFDs from end users. This system has applications to many designers and architects. It is envisioned that such a system may be used as a plugin tool for CAD applications. With the ability to concentrate less on the minutiae of CFD and more on the design task at hand the productivity of designers will be increased.

The Visualiser tool is to be made more user friendly by adding textual explanatory advice about the given visualisations. The explanatory advice would be gathered from the learning system using rule extraction and sensitivity analysis. It is envisaged that explaining the results will give users a better understanding of extra dimensionalities that are not displayable on a 3 dimensional graph.

\section{ACKNOWLEDGEMENTS}

The financial support provided by the Australian Research Council (project ID LP0455343) is gratefully acknowledged.

\section{REFERENCES}

[1] WC Becker, X Yu, J Tu, and E Lee. Single compartment fire risk analysis using a fuzzy neural network. In Proceedings of the IJCNN Conference, July 2006.

[2] GV Hadjisophocleous and N Benichou. Performance criteria used in fire safety design. Automation in Construction, 8(4):489-501, April 1999.

[3] K Jambunathan, E Lai, SL Hartle, and BL Button. Development of an intelligent front-end for a computational fluid dynamics package. Artificial Intelligence in Engineering, 6(1):27-35, 1991.

[4] B Knight and M Petridis. Flowes: An intelligent computational fluid dynamics system. Engineering Applications of Artificial Intelligence, 5(1):51-58, 1992.

[5] B Knight, S Taylor, M Petridis, J Ewer, and Galea ER. A knowledgebased system to represent spatial reasoning for fire modelling. Engineering Applications of Artificial Intelligence, 12:213-219, 1999.

[6] EWM Lee, RKK Yuen, SM Lo, KC Lam, and GH Yeoh. A novel artificial neural network fire model for prediction of thermal interface location in single compartment fire. Fire Safety Journal, (39):67-87, 2004.

[7] RL Mahajan, BM Fichera, and TW Horst. Mechanically aspirated radiation shields: A cfd and neural network design analysis. International Journal of Heat and Mass Transfer, 48(14):2856-2867, July 2005.

[8] M Petridis and B Knight. The integration of an intelligent knowledgebased system into engineering software using the blackboard structure. Advances in Engineering Software, 25:141-147, 1996.

[9] KD Steckler, JD Quitiere, and WJ Rinkinen. Flow induced by fire in a compartment. Technical report, National Bureau of Standards, Washington, DC, 1982. NBSIR 82-2520.

[10] CK Tan, SJ Wilcox, and J Ward. Use of artificial intelligence techniques for optimization of co-combustion of coal with biomass. Journal of the Energy Institute, 79(1):19-25, 2006.

[11] RKK Yuen, WM Lee, WK Kwok, CP Cheung, and GH Yeoh. Use of artificial intelligence techniques for optimization of co-combustion of coal with biomass. In International Symposium on Advances in Computational Heat Transfer, 2004 